\title{
Drug Resistance in an Organisms of Diabetic Foot Patients attending Tertiary Care Hospital in Kulasekharam
}

\author{
K. Greesh and R. Nepolean* \\ Department of Microbiology, Sree Mookambika Institute of Medical Sciences, \\ Kulesekhram, Tamilnadu, India \\ *Corresponding author
}

\section{Keywords}

Drug resistance, Diabetic foot patients, Gram negative, cephalosporins

Article Info

Accepted:

12 February 2019 Available Online: 10 March 2019

\section{A B S T R A C T}

Diabetes mellitus is a metabolic disorder characterised by chronic glycemia ${ }^{1}$ Twenty five percent diabetic patients have a risk of developing foot ulcer and limp amputation was 15$45 \%$ higher than non diabetic ulcer ${ }^{2}$. Aims are to determine the antibiotic resistance pattern of diabetic foot isolates. A total of 75 specimens (pus, swab, aspirated pus, debridement tissue) were collected from diabetic ulcer patients and the specimens processed by manually. Majority of patients $(80 \%)$ were in the age group of 51 to 70 years. The aerobic pathogens isolated were predominantely gram negative (79) and gram positive (28) bacteria. Of these 107, 70 gram negative bacilli and 20 gram positive cocci were isolated in the age group between 51 to 70 years. Of 70 gram negative bacilli Pseudomonas species was the predominant, among the gram positive cocci Staphylococcus aureus was predominant. In most of the infections in the age group between 51 to 70 years, it was polymicrobial. The antibiogram of the isolates showed that most of the Pseudomonas species was resistant to $3^{\text {rd }}$ generation cephalosporins $(74 \%)$, quinolones $(76.9 \%)$. Klebsiella species $100 \%$ resistant to amoxicillin and majority of them also resistant to $3^{\text {rd }}$ generation cephalosporins, E. coli showed high amount of resistance to amoxicillin, cefotoxime, piperacillin. Citrobacter species showed $100 \%$ resistant to amoxicillin, cefuroxime, cephalexin. Acinetobacter species showed $100 \%$ resistant to amoxicillin, $3^{\text {rd }}$ generation cephalosporins, meropenem, ciprofloxacin, gentamicin. Staphlococcus aureus $100 \%$ sensitivity to vancomycin, chloramphenicol, novobiocin.

\section{Introduction}

Diabetes mellitus is a metabolic disorder characterised by chronic hyperglycemia and about 150- 170 million people are suffering worldwide from this diseases, as per WHO reports the prevalence of diabetes will be double by 2025. Diabetes mellitus is a worldwide phenomenon, type 2 diabetes is the most common form of diabetes in developing countries like India, hence called diabetic capital of the world. In India prevalence of diabetes in rural population is about $2.4 \%$, and in urban population is about 4-11.6\%. Complications of diabetes mellitus are peripheral vascular disease, cardiovascular disease, nephropathy, retinopathy, neurological and infections. Uncontrolled 
hyperglycemia, atherosclerotic vascular disease, sensory neuropathy are the most important risk factors developing diabetic foot ulcer. ${ }^{1}$

Twenty five percent diabetic patients have a risk of developing foot ulcer and limp amputation was 15- $45 \%$ higher than non diabetic ulcer. ${ }^{2}$

\section{Pathogenesis}

For development of diabetic foot ulcer, the most important risk factors are peripheral neuropathy and impaired blood circulation from peripheral vascular disease., 3,5 In diabetes mellitus patients, one of the major complications is diabetic ulcer. Fifteen percent diabetes mellitus patients develop diabetic foot ulcer and leads to $84 \%$ of foot amputation. ${ }^{6}$

\section{Neuropathy}

Development of neuropathy is as a result of hyperglycemia induced metobolic disorder. The most important one is polyol pathway. Hyperglycemia state will favour aldose reductase and sorbitol dehydrogenase which will convert intracellular glucose to sorbitol and fructose and due to the accumulation of these sugar products leads to decrease in the synthesis of myoinositol, which is needed for normal neuron conduction. The conversion of glucose leads to depletion of nicotinamide adenine dinucleotide phosphate which is required for detoxification of reactive oxygen species and for synthesis of vasodilator nitric oxide. This leads to oxidative stress on nerve cells and increase vasoconstricton leads to ischaemia, which result will nerve cell injury and death. This also contributes to abnormal glycation of nerve cells and leads to inappropriate action of protein kinase $\mathrm{C}$ and leads to further nerve damage. In diabetic patients neuropathy develop in motor, sensory, autonomic components of nervous system. Imbalance between flexion and extension due to damage of innervatons of intrinsic foot muscles, leads to foot deformities that create abnormal bony prominence and pressure points, which favour for skin break down and ulceration.

Autonomic neuropathy leads to suppression of the function of sweat and oil gland. Foot loses natural function of the moisturising the skin and becomes dry which leads breakdown and gradually develop infection.

Sensory neuropathy wounds are unnoticed by the patients which worseness and exacerbates the development of ulcer.

\section{Vascular disease}

The persistent hyperglycemic state leads to endothelial cell dysfunction and smooth cell abnormalities in peripheral arteries which result in the decreases of endothelium derived vasodilator that leads to vasoconstriction. The diabetes hyperglycemic state leads to increase in thromboxane A2, a vasoconstrictor, platelet aggregation which promote the risk for hypercoagulability, and alteration in the vascular extra cellular matrix leads arterial lumen stenosis. The other factors like smoking, hypertension, hyperlipidemia contribute to the development of peripheral arterial disease. $^{3}$

The present study was carried out to determine the aerobic bacterial isolates cultured from diabetic foot infections and their susceptibility to commonly used antibiotics.

\section{Materials and Methods}

The study was conducted in a tertiary care hospital at Kulasekharam from june 2014 to August 2015. The study was approved by the Institutional Ethical and Research committee. 
A total of 75 specimens (pus, swabs, aspirated pus, debridement tissue) were collected from diabetic ulcer patients ${ }^{7}$. The samples were collected in dressing room for out patients and in wards for inpatient and then immediately transported to the laboratory and the specimens were processed without any delay by manually $8,9,10$.

Phenotypic screening of ESBL detection with combination disk method (ceftazidime (30microgram) /ceftazidime (30microgram) and clavulinic acid (10 microgram) \& cefotaxime (30microgrm)/ cefotaxime (30 microgram)/and clvulinic acid (10 microgram). ${ }^{2,11}$

Inclusion criteria: Patient admitted with clinically diagnosed diabetes mellitus, supported by laboratory findings and presented with ulcer.

Exclusion criteria: Patient with ulcers, who are not diabetic proved by clinical or laboratory investigations \& Gestational diabetes mellitus with ulcer.

\section{Results and Discussion}

The age group varied from 41 to 90 years. Majority of patients $(80 \%)$ between 41 to 70 years (Table 1). The predominant pathogens isolated were gram negative bacilli constituting $73.8 \%$ and gram positive cocci constituting 26, 2\%. Among the gram negative bacilli Pseudomonas species was predominant having $36.2 \%$ incidence and among the gram positive cocci Staphylococcus aureus were the predominant pathogens accounting $42.8 \%$ (Fig. 1). Pseudomonas species shows resistant to cefotoxime (100\%), ciprofloxacin $(77 \%)$, gentamicin $(72 \%)$, piperacillin $(71 \%)$, ceftazidime (61\%), cefoperazone (61\%), meropenem $(46 \%$. Sensitivity to piperacillin/tazobactum $(68 \%$, netimicin (66\%), amikacin (52\%) (Table 2). Klebsiella species resistant to amoxicillin (100\%), cefolexin (80\%), ceftozidime (80\%), cefotoxime $(79 \%)$, cefuroxime $(69 \%)$, cotrimoxazole (67\%), ciprofloxacin (67\%), gentamicin $(50 \%)$. Sensitivity to meropenem (91\%), netilmicin (79\%), amikacin (69\%), piperacillin $(65 \%)$ (Table 2). Proteus species shows resistant to cefolexin (80\%), amoxicillin (79\%), cotrimoxazole (67\%), cefotoxime (62\%), cefoperazone (57\%). sensitivity to piperacillin/tazobactum (87\%), meropenem (85\%), piperacillin $(64 \%)$, netilmicin (64\%) (Table 3). E. coli was $89 \%$ resistant to amoxicillin, piperacillin, cefotoxime and cefolexin $(78 \%)$, cefoperazone (71\%), cefuroxime (67\%), ciprofloxacin (67\%), piperacillin/tazobactum (63\%), cotrimoxazole $(50 \%)$. Sensitivity to netilmicin (86\%), amikacin (78\%), meropenem (67\%), gentamicin (67\%) (Table 3). Citrobacter species was $100 \%$ resistant to amoxicillin, cefolexin, cefuroxime and cefotoxime $(83 \%)$, ceftazidime $(83 \%)$. sensitivity to netilmicin was $(100 \%)$, cotrimoxazole $(83 \%)$, amikacin (80\%) meropenem (67\%) (Table 4). Enterobacter species shows $100 \%$ sensitivity to amoxicillin, piperacillin/tazobactum, netilmicin, gentamicin, ciprofloxacin, cotrimoxazole, cefolexin, ceftazidime, cefuroxime, cefotoxime, cefoperazone, meropenem (Table 4). Acinetobacter species was $100 \%$ resistant to amoxicillin, piperacillin/tazobactum, piperacillin, ciprofloxacin, cefolexin, cefotoxime, cefuroxime, gentamicin, meropenem. Sensitivity to amikacin (40\%) (Table 5). Staphylococcus aureus shows $100 \%$ sensitivity to vancomycin, novobiocin, chloramphenicol and clindamycin (92\%), teicoplanin $(90 \%)$, cefoxitin $(83 \%)$, netilmicin (78\%). Resistant to penicillin (91\%), cotrimoxazole $(81 \%)$ and erythromycin (69\%). CONS was resistant to erythromycin (100\%), penicillin (83\%), cotrimoxazole (67\%), ciprofloxacin (60\%) and sensitivity to vancomycin, novobiocin and chloramphenicol 
was $100 \%$ respectively, teicoplanin $(80 \%)$, gentamicin $(75 \%)$, netilmicin $(60 \%)$ (Table 6). Streptococcus pyogenes shows $100 \%$ sensitivity to penicillin, netilmicin, cefuroxime, cefotoxime and amikacin (75\%), ciprofloxacin (75\%), tetracycline (66\%) (Table 7). Streptococcus species shows 100\% sensitivity to penicillin, amikacin, netilmicin, vancomycin, and $50 \%$ resistant to tetracycline, cotrimoxazole, ciprofloxacin.

Enterococcus species shows $100 \%$ sensitive to ciprofloxacin, cefotoxime, cefuroxime, vancomycin and $50 \%$ resistant to tetracycline, amikacin, netilmicin, cotrimoxazole (Table 8). Among the gram negative bacilli $67 \%$ of E.coli, $47 \%$ of Klebsiella, $17 \%$ of Citrobacter species, $15 \%$ of Pseudomonas species, $13 \%$ of Proteus species were ESBL producers (Table 9 and Fig. 2).

This study presents clinical and microbiological profile of Diabetic foot ulcers. About 150 to 170 million populations are suffering from diabetes mellitus worldwide. ${ }^{1}$ In India nearly 40 million people are diabetics and their socioeconomic status is poor.

Diabetic foot infections are seen in $20 \%$ of the patients and hence are the most commonly faced clinical problem. Ulcers treated inappropriately may lead to amputation or disarticulation in varying levels atleast once in such patients' life time. ${ }^{12}$

This study was carried out at SMIMS, Kulasekharam from June 2014 to August 2015. Samples (swabs, aspirated pus, debrided tissue) from 75 patients of diabetic foot ulcer was collected after receiving written consent from the patient. Majority of patients $(80 \%)$ were in the age group of 51 to 70 years. The aerobic pathogens isolated were predominantly gram negative (79) followed by gram positive (28) bacteria (Fig. 1). Of these 107, 70 gram negative bacilli and 20 gram positive cocci were isolated in the age group between 51 to 70 years.

Of 70 gram negative bacilli isolated in the age group of 51 to 70 years, Pseudomonas species was the predominant isolate followed by Klebsiella species, Proteus species, E.coli, Citrobacter species, Acinetobacter species and Enterobacter species.

Among the gram positive cocci organisms isolated in this age group (51 to 70years), Staphylococcus aureus was the predominant pathogen followed by coagulase negative Staphylococci, Streptococci pyogenes, other beta hemolytic Streptococci, Streptococcus species and only one Enterococcus species was isolated in this age group. A evidenced by Shanmugam.P et al., Pappu.K et al., also have shown the similar findings. 8,13 .

In most of the infections in the age group between 51 to 70 years it was polymicrobial (31 cases), whereas monomicrobial etiology was seen in 27 cases. As also reported by Chopdekar et al., 2011. ${ }^{14}$ There was no growth in two clinical samples this could be due to the prior antibiotic therapy before coming to the hospital or could be anerobic organisms the isolation of which was not attempted in the study.

The antibiogram of the isolates showed that most of the Pseudomonas species was resistant to $3^{\text {rd }}$ generation Cephalosporins (74\%) followed by Quinolones (76.9\%) (Table $2)$. Twenty five $(68.4 \%)$ isolates were sensitive to Piperacillin/Tazobactam followed by Netilmicin $(65.8 \%)$ and Meropenem (53.8\%) Gentamicin (74\%). Shanmugam et al., (2013) in their study also have shown, Pseudomonas being $50 \%$ resistant to Gentamicin and Quinolones, 61\% resistant to $3^{\text {rd }}$ generation Cephalosporin but $100 \%$ resistant to Meropenem. But in our study resistant to Meropenem was $46.2 \%$. 
Table.1 Distribution of age

\begin{tabular}{|c|c|c|}
\hline Age & Patients & Percentage \\
\hline 41 to 50 & 8 & 11 \\
\hline 51 to 60 & 30 & 40 \\
\hline 61 to 70 & 30 & 40 \\
\hline 71 to 80 & 6 & 8 \\
\hline 81 to 90 & 1 & 1 \\
\hline
\end{tabular}

The age group varied from 41 to 90 years (Table 1 )

Table.2 Antibiogram

\begin{tabular}{|l|l|l|l|l|l|l|}
\hline & \multicolumn{3}{c}{ SSEUDOMONAS SP } & \multicolumn{2}{c|}{ KLEBSIELLA SP } & \\
\hline & Sensitive & Intermediate & Resistant & Sensitive & Intermediate & Resistant \\
\hline PENICILLIN & - & - & - & - & - & - \\
\hline AMOXYCILLIN & - & - & - & $0.00 \%$ & $0.00 \%$ & $100 \%$ \\
\hline TETRACYCLINE & & & & & & \\
\hline AMIKACIN & $52.17 \%$ & $8.69 \%$ & $39.13 \%$ & $69.23 \%$ & $0.00 \%$ & $30.76 \%$ \\
\hline $\begin{array}{l}\text { PIPERACILLIN/TAZOB } \\
\text { ACTUM }\end{array}$ & $68.42 \%$ & $0.00 \%$ & $31.58 \%$ & $42.85 \%$ & $21.42 \%$ & $35.71 \%$ \\
\hline NETILMICIN & & & & & & \\
\hline PIPERACILLIN & $28.83 \%$ & $15.38 \%$ & $19.23 \%$ & $78.57 \%$ & $0.00 \%$ & $21.43 \%$ \\
\hline CIPROFLOXACIN & $23.07 \%$ & $0.00 \%$ & $71.43 \%$ & $6.66 \%$ & $28.34 \%$ & $65 \%$ \\
\hline COTRIMOXAZOLE & & & $76.92 \%$ & $25.25 \%$ & $8.33 \%$ & $66.66 \%$ \\
\hline MEROPENEM & $53.85 \%$ & $0.00 \%$ & $46.15 \%$ & $90.91 \%$ & $0.00 \%$ & $9.09 \%$ \\
\hline GENTAMYCIN & $24 \%$ & $4 \%$ & $72 \%$ & $50 \%$ & $0.00 \%$ & $50 \%$ \\
\hline VANCOMYCIN & - & - & - & & & \\
\hline CEFOLEXIN & & & & $20.00 \%$ & $0.00 \%$ & $80.00 \%$ \\
\hline CEFUROXIME & & & & $23.08 \%$ & $7.69 \%$ & $69.23 \%$ \\
\hline CEFOTOXIME & $0.00 \%$ & $0.00 \%$ & $100.00 \%$ & $21.43 \%$ & $0.00 \%$ & $78.57 \%$ \\
\hline CEFOPERAZONE & $30.7 \%$ & $7.69 \%$ & $61.5 \%$ & $20 \%$ & $13.33 \%$ & $66.66 \%$ \\
\hline CEFTAZIDIME & $33.33 \%$ & $5.56 \%$ & $61.11 \%$ & $20 \%$ & $0.00 \%$ & $80 \%$ \\
\hline
\end{tabular}

Seventy four percent of Pseudomonas species was resistant to $3^{\text {rd }}$ generation Cephalosporins. (Table:2) 
Table.3 Antibiogram

\begin{tabular}{|l|l|l|l|l|l|l|}
\hline & \multicolumn{3}{|c|}{ PROTEUS SPECIES } & \multicolumn{3}{c|}{ E.COLI } \\
\hline & Sensitive & Intermediate & Resistant & Sensitive & Intermediate & Resistant \\
\hline PENICILLIN & - & - & - & & & \\
\hline AMOXYCILLIN & $21.42 \%$ & $0.00 \%$ & $78.57 \%$ & $11 \%$ & $0.00 \%$ & $89.00 \%$ \\
\hline TEICOPLANIN & - & - & - & - & - & - \\
\hline CLINDAMYCIN & - & - & - & - & - & - \\
\hline ERYTHROMYCIN & - & - & - & - & - & - \\
\hline AMIKACYCLINE & - & - & - & & & \\
\hline PIPERACINLIN/TAZOBACTAM & $64.28 \%$ & $0.00 \%$ & $35.71 \%$ & $78 \%$ & $0.00 \%$ & $22 \%$ \\
\hline NETILMICIN & $86.66 \%$ & $13.33 \%$ & $0.00 \%$ & $37.50 \%$ & $0.00 \%$ & $62.50 \%$ \\
\hline PIPERACILLIN & $63.63 \%$ & $0.00 \%$ & $36.36 \%$ & $86 \%$ & $0.00 \%$ & $14 \%$ \\
\hline CIPROFLOXACIN & $63.64 \%$ & $0.00 \%$ & $36.36 \%$ & $11 \%$ & $0.00 \%$ & $89 \%$ \\
\hline COTRIMOXAZOLE & $40 \%$ & $13 \%$ & $47.00 \%$ & $33 \%$ & $0.00 \%$ & $67 \%$ \\
\hline MEROPENEM & $33 \%$ & $0.00 \%$ & $67 \%$ & $50 \%$ & - & $50 \%$ \\
\hline GENTAMICIN & $84.62 \%$ & $7.69 \%$ & $7.69 \%$ & $67 \%$ & $11 \%$ & $22 \%$ \\
\hline VANCOMYCIN & $53 \%$ & $0.00 \%$ & $47 \%$ & $67 \%$ & $0.00 \%$ & $33 \%$ \\
\hline CEFOLEXIN & - & - & - & - & - & - \\
\hline CEFUROXIME & $20 \%$ & $0.00 \%$ & $80 \%$ & $22 \%$ & $0.00 \%$ & $78 \%$ \\
\hline CEFOTOXIME & $27 \%$ & $0.00 \%$ & $73 \%$ & $33 \%$ & $0.00 \%$ & $67 \%$ \\
\hline CEFOPERAZONE & $30.77 \%$ & $7.69 \%$ & $61.54 \%$ & $11 \%$ & $0.00 \%$ & $89 \%$ \\
\hline CEFTAZIDIME & $35.71 \%$ & $7.14 \%$ & $57.14 \%$ & $29 \%$ & $0.00 \%$ & $71 \%$ \\
\hline
\end{tabular}

shows Proteus species $87 \%$ sensitivity to Piperacillin/Tazobactam, $85 \%$ to Meropenem and E.coli shows 89\% resistant to Amoxicillin, Piperacillin, Cefotoxime

Table.4 Antibiogram

\begin{tabular}{|c|c|c|c|c|c|c|}
\hline & \multicolumn{2}{|c|}{ CITROBACTER SPECIES } & \multirow[b]{2}{*}{ Resistant } & \multicolumn{2}{|c|}{ ENTEROBACTER SPECIES } & \multirow[b]{2}{*}{ Resistant } \\
\hline & Sensitive & Intermediate & & Sensitive & Intermediate & \\
\hline AMOXYCILLIN & $0.00 \%$ & $0.00 \%$ & $100.00 \%$ & $100.00 \%$ & & \\
\hline \multicolumn{7}{|l|}{ TETRACYCLINE } \\
\hline PIPERACILLIN/TAZOBACTUM & $17 \%$ & $17 \%$ & $67 \%$ & $100.00 \%$ & & \\
\hline NETILMICIN & $100 \%$ & $0.00 \%$ & $0.00 \%$ & $100.00 \%$ & & \\
\hline COTRIMOXAZOLE & $83 \%$ & $0.00 \%$ & $17 \%$ & $100.00 \%$ & & \\
\hline MEROPENEM & $67 \%$ & $0.00 \%$ & $33 \%$ & $100.00 \%$ & & \\
\hline GENTAMYCIN & $25.00 \%$ & $25.00 \%$ & $50.00 \%$ & $100.00 \%$ & & \\
\hline CEFOLEXIN & $0.00 \%$ & $0.00 \%$ & $100.00 \%$ & $100.00 \%$ & & \\
\hline CEFUROXIME & $0.00 \%$ & $0.00 \%$ & $100.00 \%$ & $100.00 \%$ & & \\
\hline
\end{tabular}

Table.4 shows Citrobacter and Enterobacter species shows 100\% sensitivity to Netilmycin 
Table.5 Antibiogram

\begin{tabular}{|l|c|c|c|}
\hline & \multicolumn{2}{|c|}{ Acinetobacter SP } & \\
\hline & Sensitive & Intermediate & Resistant \\
\hline PENICILLIN & & & \\
\hline AMOXYCILLIN & $0.00 \%$ & $0.00 \%$ & $100.00 \%$ \\
\hline TEICOPLANIN & - & - & - \\
\hline CLINDAMYCIN & - & - & - \\
\hline ERYTHROMYCIN & - & - & - \\
\hline TETRACYCLINE & & & \\
\hline AMIKACIN & $40.00 \%$ & $0.00 \%$ & $60.00 \%$ \\
\hline PIPERACILLIN/TAZOBACTAM & $0.00 \%$ & $0.00 \%$ & $100.00 \%$ \\
\hline NETILMICIN & $40.00 \%$ & $0.00 \%$ & $60.00 \%$ \\
\hline PIPERACILLIN & $0.00 \%$ & $0.00 \%$ & $100.00 \%$ \\
\hline CIPROFLOXACIN & $0.00 \%$ & $0.00 \%$ & $100.00 \%$ \\
\hline COTRIMOXAZOLE & $20.00 \%$ & $40.00 \%$ & $40.00 \%$ \\
\hline MEROPENEM & $0.00 \%$ & $0.00 \%$ & $100.00 \%$ \\
\hline GENTAMICIN & $0.00 \%$ & $0.00 \%$ & $100.00 \%$ \\
\hline CEFOLEXIN & $0.00 \%$ & $0.00 \%$ & $100.00 \%$ \\
\hline CEFUROXIME & $0.00 \%$ & $0.00 \%$ & $100.00 \%$ \\
\hline CEFOTOXIME & $0.00 \%$ & $0.00 \%$ & $100.00 \%$ \\
\hline CEFOPERAZONE & $0.00 \%$ & $0.00 \%$ & $100.00 \%$ \\
\hline CEFTAZIDIME & $0.00 \%$ & $0.00 \%$ & $100.00 \%$ \\
\hline
\end{tabular}

Table.5 Acinetobacter species 100\% resistant to Amoxicillin, Piperacillin/Tazobactam, Piperacillin, Ciprofloxacin, Meropenem, Cefotoxime, Cefuroxime, Cefolexin, Gentamicin

Table.6 Antibiogram

\begin{tabular}{|c|c|c|c|c|c|c|}
\hline & \multicolumn{2}{|c|}{ S. aureus } & \multicolumn{3}{c|}{ CONS } & \multicolumn{2}{|c|}{ Resistant } \\
\hline & Sensitive & Intermediate & Resistant & Sensitive & Intermediate & R \\
\hline PENICILLIN & $9.00 \%$ & $0.00 \%$ & $91.00 \%$ & $0.00 \%$ & $17.00 \%$ & $83.00 \%$ \\
\hline AMOXYCILLIN & & & & - & - & - \\
\hline TEICOPLANIN & $90.00 \%$ & $10.00 \%$ & $0.00 \%$ & $80.00 \%$ & $0.00 \%$ & $20.00 \%$ \\
\hline CLINDAMYCIN & $91.67 \%$ & $0.00 \%$ & $8.33 \%$ & $60.00 \%$ & $0.00 \%$ & $40.00 \%$ \\
\hline ERYTHROMYCIN & $15.38 \%$ & $15.38 \%$ & $69.23 \%$ & $0.00 \%$ & $0.00 \%$ & $100.00 \%$ \\
\hline TETRACYCLINE & $58.00 \%$ & $17.00 \%$ & $25.00 \%$ & $50.00 \%$ & $0.00 \%$ & $50.00 \%$ \\
\hline AMIKACIN & $63 \%$ & $25 \%$ & $12.5 \%$ & $50.00 \%$ & $16.67 \%$ & $33.33 \%$ \\
\hline PIPERACILLIN/TAZOBACTAM & & & & & & \\
\hline NETILMICIN & $78 \%$ & $0.00 \%$ & $22 \%$ & $60.00 \%$ & $0.00 \%$ & $40.00 \%$ \\
\hline PIPERACILLIN & & & & & & \\
\hline CIPROFLOXACIN & $33 \%$ & $0.00 \%$ & $67 \%$ & $40.00 \%$ & $0.00 \%$ & $60.00 \%$ \\
\hline COTRIMOXAZOLE & $19.00 \%$ & $0.00 \%$ & $81.00 \%$ & $33.33 \%$ & $0.00 \%$ & $66.67 \%$ \\
\hline MEROPENEM & & & & & & \\
\hline GENTAMICIN & $40.00 \%$ & $20.00 \%$ & $40.00 \%$ & $75.00 \%$ & $0.00 \%$ & $25.00 \%$ \\
\hline VANCOMYCIN & $100.00 \%$ & $0.00 \%$ & $0.00 \%$ & $100.00 \%$ & $0.00 \%$ & $0.00 \%$ \\
\hline NOVOBIOCIN & $100.00 \%$ & $0.00 \%$ & $0.00 \%$ & $100.00 \%$ & $0.00 \%$ & $0.00 \%$ \\
\hline CHOLORAMPHENICOL & $100.00 \%$ & $0.00 \%$ & $0.00 \%$ & $100.00 \%$ & $0.00 \%$ & $0.00 \%$ \\
\hline CEFOXITIN & $83.33 \%$ & $0.00 \%$ & $16.67 \%$ & $50.00 \%$ & $0.00 \%$ & $50.00 \%$ \\
\hline CEFOTOXIME & & & & - & - & - \\
\hline CEFOPERAZONE & & & & & & \\
\hline CEFTAZIDIME & & & & & & \\
\hline
\end{tabular}

Table.6 Eight one percentage of Staphylococcus aureus was resistant to cotrimaxozole in our study followed by Erythromycin (69\%), Ciprofloxacin (67\%), Gentamicin (40\%) 
Table.7 Antibiogram

\begin{tabular}{|c|c|c|c|c|c|c|}
\hline & \multicolumn{2}{|c|}{ Streptococcus pyogenes } & \multirow[b]{2}{*}{ Resistant } & \multicolumn{2}{|c|}{$\begin{array}{l}\text { BETA - HEMOLYTIC } \\
\text { Streptococcus }\end{array}$} & \multirow[b]{2}{*}{ Resistant } \\
\hline & Sensitive & Intermediate & & Sensitive & Intermediate & \\
\hline PENICILLIN & $100.00 \%$ & $0.00 \%$ & $0.00 \%$ & 100.00 & $0.00 \%$ & $0.00 \%$ \\
\hline AMOXYCILLIN & & & & - & - & - \\
\hline TEICOPLANIN & - & - & - & - & - & - \\
\hline CLINDAMYCIN & - & - & - & - & - & - \\
\hline ERYTHROMYCIN & - & - & - & - & - & - \\
\hline TETRACYCLINE & $66.67 \%$ & $0.00 \%$ & $33.33 \%$ & $50.00 \%$ & $0.00 \%$ & $50.00 \%$ \\
\hline AMIKACIN & $75.00 \%$ & $0.00 \%$ & $25.00 \%$ & $100.00 \%$ & $0.00 \%$ & $0.00 \%$ \\
\hline \multicolumn{7}{|c|}{ PIPERACILLIN/TAZOBACTAM } \\
\hline NETILMICIN & $100.00 \%$ & $0.00 \%$ & $0.00 \%$ & $100.00 \%$ & $0.00 \%$ & $0.00 \%$ \\
\hline \multicolumn{7}{|l|}{ PIPERACILLIN } \\
\hline CIPROFLOXACIN & $75.00 \%$ & $0.00 \%$ & $25.00 \%$ & $50.00 \%$ & $0.00 \%$ & $50.00 \%$ \\
\hline \multicolumn{7}{|l|}{ COTRIMOXAZOLE } \\
\hline \multicolumn{7}{|l|}{ MEROPENEM } \\
\hline \multicolumn{7}{|l|}{ GENTAMYCIN } \\
\hline CEFUROXIME & $100 \%$ & $0.00 \%$ & $0.00 \%$ & & & \\
\hline CEFOTOXIME & $100.00 \%$ & $0.00 \%$ & $0.00 \%$ & $100.00 \%$ & $0.00 \%$ & $0.00 \%$ \\
\hline
\end{tabular}

Streptococcus pygenes and other beta hemolytic streptococci were $100 \%$ sensitive to penicillin (100\%). However Streptococcus pygenes was also sensitive to Netilmicin, Cefotoxime and Cefuroxime (100\%) as showed in Table:7

Table.8 Antibiogram

\begin{tabular}{|c|c|c|c|c|c|c|}
\hline & \multicolumn{2}{|c|}{ Enterococcus sp } & \multirow[b]{2}{*}{ Resistant } & \multicolumn{3}{|c|}{ Streptococcus SP } \\
\hline & Sensitive & Intermediate & & Sensitive & Intermediate & Resistant \\
\hline PENICILLIN & & & & $100 \%$ & & \\
\hline AMOXYCILLIN & - & - & - & & & \\
\hline TEICOPLANIN & - & - & - & & & \\
\hline CLINDAMYCIN & - & - & - & & & \\
\hline ERYTHROMYCIN & - & - & - & & & \\
\hline TETRACYCLINE & $50.00 \%$ & $0.00 \%$ & $50.00 \%$ & $50.00 \%$ & & $50.00 \%$ \\
\hline AMIKACIN & $50.00 \%$ & $0.00 \%$ & $50.00 \%$ & $100.00 \%$ & & $0.00 \%$ \\
\hline PIPERACILLIN/TAZOBACTUM & - & - & - & & & \\
\hline NETILMICIN & $50.00 \%$ & $0.00 \%$ & $50.00 \%$ & $100.00 \%$ & $0.00 \%$ & $0.00 \%$ \\
\hline PIPERACILLIN & - & - & - & & & \\
\hline CIPROFLOXACIN & $100.00 \%$ & $0.00 \%$ & $0.00 \%$ & $50.00 \%$ & $0.00 \%$ & $50.00 \%$ \\
\hline COTRIMOXAZOLE & $50.00 \%$ & $0.00 \%$ & $50.00 \%$ & $50.00 \%$ & $0.00 \%$ & $50.00 \%$ \\
\hline MEROPENEM & - & - & - & & & \\
\hline GENTAMYCIN & - & - & - & & & \\
\hline VANCOMYCIN & $100.00 \%$ & $0.00 \%$ & $0.00 \%$ & $100.00 \%$ & $0.00 \%$ & $0.00 \%$ \\
\hline CEFUROXIME & $100.00 \%$ & $0.00 \%$ & $0.00 \%$ & & & \\
\hline CEFOTOXIME & $100.00 \%$ & $0.00 \%$ & $0.00 \%$ & & & \\
\hline
\end{tabular}

Table. 8 shows Enterococcus species $100 \%$ sensitive to ciprofloxacin, cefotoxime, cefuroxime and Vancomycin 
Table.9 ESBL

\begin{tabular}{|c|c|}
\hline KLEBSIELLA SP & $47 \%$ \\
\hline E.COLI & $67 \%$ \\
\hline PSEUDOMONAS SP & $15 \%$ \\
\hline PROTEUS SP & $13 \%$ \\
\hline CITROBACTER SP & $17 \%$ \\
\hline
\end{tabular}

Table. 9 shows E. coli was the dominant ESBL producer (67\%)

Fig.1 List of organisms: The predominant pathogens isolated were gram negative bacilli constituting $73.8 \%$ and gram positive cocci constituting 26.2\%. Among the gram negative bacilli Pseudomonas species was predominant having $36.2 \%$ incidence and among the gram positive cocci Staphylococcus aureus were the predominant pathogens accounting $42.8 \%$

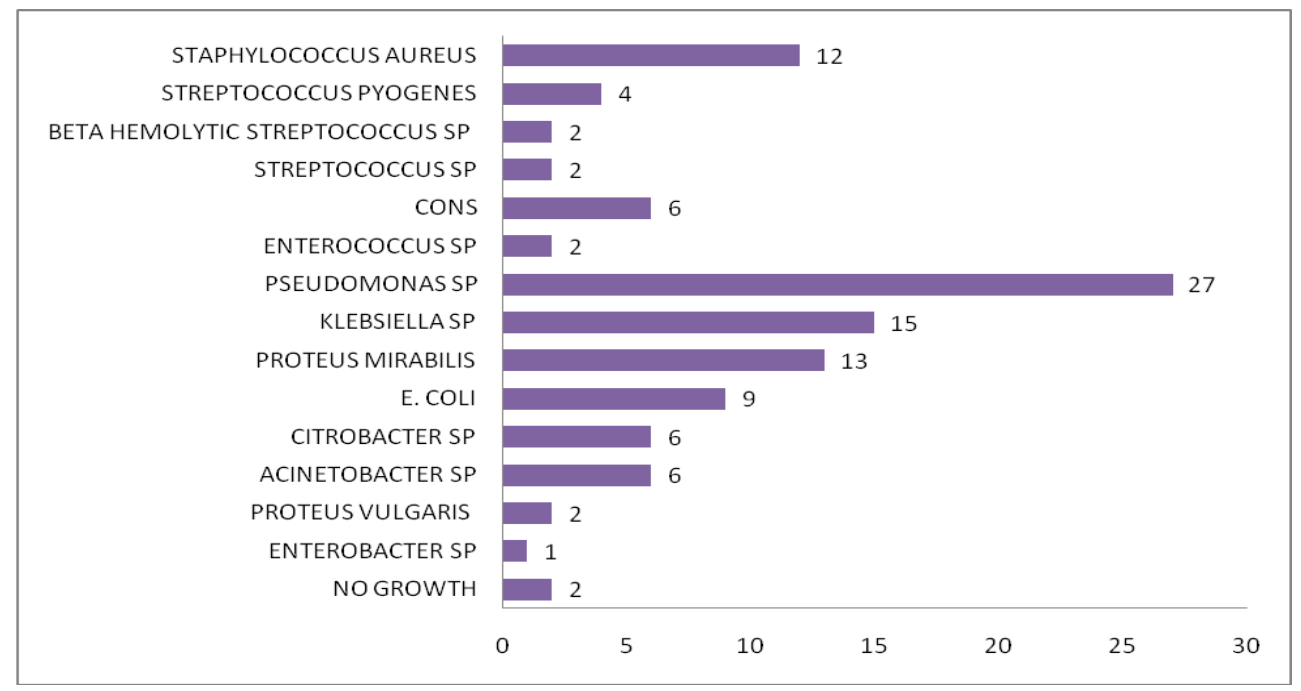

Fig.2 List of organisms Producing ESBL

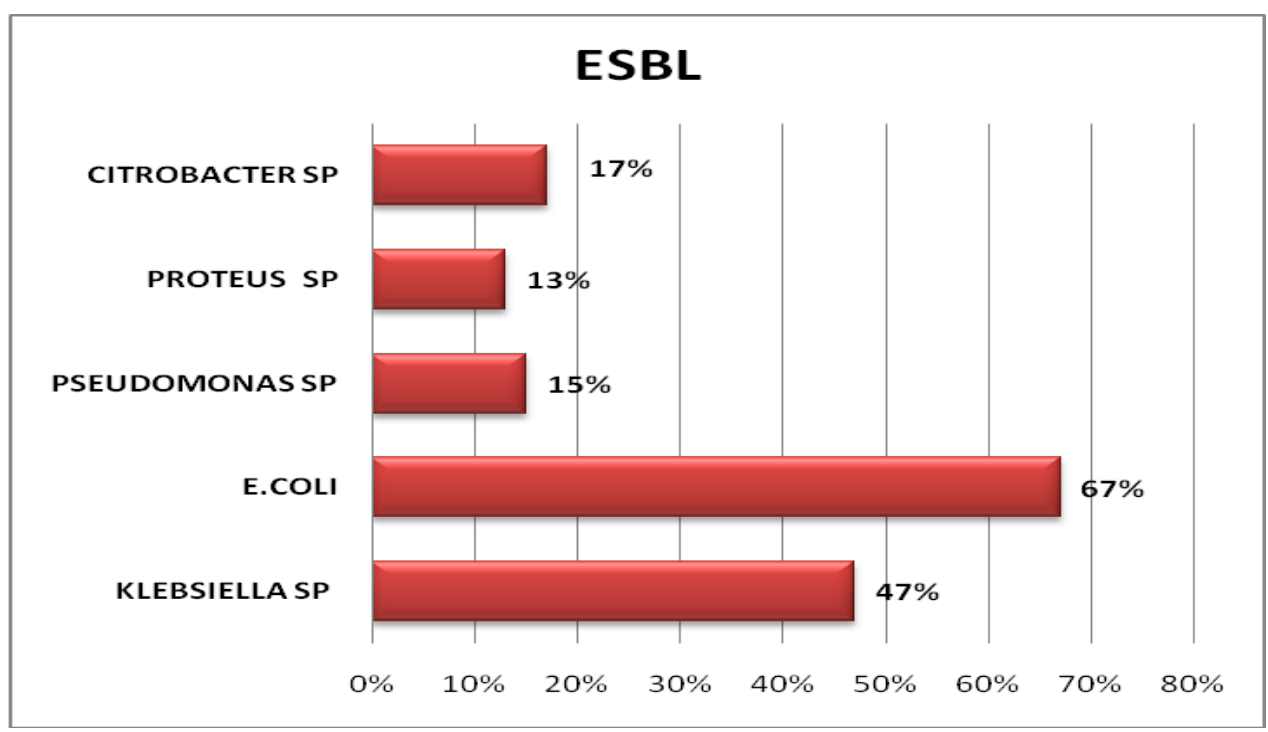


It is surprising to note that high amount resistant to Meropenem which could be probably due to use of Carbapenems prescribed by general practitioners which must have resulted in developing resistance to Mereopenem before coming to our hospital.

Klebsiella isolates were $100 \%$ resistant to Amoxicillin. Majoritry of them were also resistant to $3^{\text {rd }}$ generation Cephalosporin but $91 \%$ of the isolates were sensitive to Meropenem followed by Netilmicin (78.5\%), Amikacin (69.2\%), Gentamycin (50\%). Majority of Proteus species showed sensitivity to Piperacillin/Tazabactam (86.7\%) followed by Meropenem (84.6\%). E.coli showed high amount of resistance to Amoxicillin, Cefotoxime, Piperacillin. However majority of orgainsms were sensitive to Netilmicin (86\%), Amikacin (78\%) followed by Gentamicin and Meropenem (67\% each).

Citrobacter species showed $100 \%$ resistant to Amoyxicillin, Cefuroxime, Cephalexin.However they were $100 \%$ sensitive to Amikacin and Netilmicin.

Enterobacter species showed $100 \%$ sensitivity to most of the antibiotic used.

Acinetobacter species showed $100 \%$ resistant to Amoxicillin, all the $3^{\text {rd }}$ generation Cephalosporins and Meropenem, Ciprofloxacin and Gentamicin. Only Amikacin and Netilmicin showed $40 \%$ sensitivity.

Among gram positive organisms Staphylococcus aureus was the predominant pathogen and the antibiogram showed $100 \%$ sensitivity to Vancomycin, Choloramphenicol and Novabiocin followed by Clindamycin $(91.7 \%)$ and Teicoplanin (90\%). Of the total 18 Staphylococcus aureus, 2 were MRSA. Kaur.N et al., 2014, ${ }^{15}$ also showed less sensitivity to Clindamycin is contrast to our study.

CONS showed a similar pattern of sensitivity to Vancomycin, Novobiocin, Choloramphenicol as Staphylococcus aureus. However they were less sensitive to Clindamycin (Table 6). One Coagulase negative Staphylococcus was resistant to Methicillin. Paul.S et al., 2009, ${ }^{56}$ found that $8.7 \%$ of Methicillin Resistant in their study.

All the Streptococcus pyogens were sensitive to Penicillin, they were also $100 \%$ sensitive to Netilmicin, Cefotoxime and Cefuroxime (Table 7) Enterococcus showed 100\% sensitivity to Vancomycin, Cefuroxime, Cefotoxime. Ciprofloxacin seems to be good antibiotic for treating infections with Enterococcus species, since they showed $100 \%$ sensitivity in our study.

Extended spectrum beta lactamase producing organisms were mainly seen in E.coli (67\%), Klebsiella (47\%). However ESBL was not a major problem in Pseudomonas species, Proteus species, Citrobacter species. However AmpC, MBL were not looked for in our study (Table 9).

In conclusion,

$>\quad$ Diabetic foot ulcer infection should be treated according to culture and sensitivity report.

$>\quad$ To avoid unnecessary usage of antibiotic which may result in development of Multi Drug Resistant strains.

$>\quad$ Emprical treatment should be based on recent report of articles of same geographical region.

$>\quad$ Sensitivity pattern varies from place to place. This study could help clinician to know the sensitivity pattern of organism.

$>\quad$ Diabetic foot ulcer treatment should be based on multidisciplinary approach. 
It is health providers responsibility to enlighten the foot care in diabetes and consequences of foot infection and use of Proper foot wear which could decrease development of foot ulcer.

\section{References}

1. WHO 1998, Prevention and control of Diabetes Mellitus, Report of an Inter country workshop, Dhaka, Bangladesh, 27- 30, SEA/NCD/40.

2. Esmat. M.M, Islam. A.S, Diabetic foot infection bacteriological causes and antimicrobial therapy. J American Sci 2012; 8(10): 389-93.

3. Clayton. W, Jr, Tom. A, 2009. A review of the pathophysiology, classification and treatment of foot ulcers in diabetic patients. Clinidia J Org; 27: 52-8.

4. Obayashi. K, Akmatsu. H, 2005. Exogenous nitric oxide enhances the synthesis type I collagen and heat shock protein 47 by normal human dermal fibroblasts. J Derm Scien; 41(2): 121-26.

5. Ravanthi. L, Kahari. V.M, 2000 Matrix metalloproteinases in wound repair. Int $\mathbf{J}$ of Mole Med; 6 (4): 391-407.

6. Brem. H, Canic. M.T, 2007 Cellular and molecular basis of wound healing in diabetes. J of ClinInves; 117(5): 1219222.

7. Sheahan. M.G, Sheahan. C.M, 2012. Minor Amputations. In: Fischer JE. Fischer's Mastery of Surgery. Vol $2.6^{\text {th }}$ ed. New Delhi publ: Wolters Kluwer India; Pp.2305-12.
8. Shanmugam. P, Jeya. M, 2013. The Bacteriology of Diabetic Foot Ulcers with a special reference to multidrug resistant strains. J Clin Diagnostic Res; 7(3): 44145.

9. Duguid. J.P, Collee JG, 1996. Mackie and McCartney Practical Medical Microbiology. $14^{\text {th }}$ ed. New York: Churchill Livingstone; Pp. 793-800.

10. Collee. J.G, Marr. W, 1996. Specimen collection, culture container, media. In: Mackie and McCartney Practical Medical Microbiology. $14^{\text {th }}$ ed. New York: Churchill Livingstone. Pp. 95-111.

11. Miles. R.S, Amyes. S.G.P, 1996. Laboratory control of antimicrobial therapy. Mackie and McCartney Practical Medical Microbiology. $14^{\text {th }}$ ed. New York: Churchill Livingstone; p.151-77.

12. Banashankari. G.S, Rudresh. H.K, 2012. Prevalence of gram negative bacteria in diabetic foot A clinic microbiological study. Al Ameen J Med Sci; 5(3): 224-32.

13. Pappu. A.K, Sinha. A, 2011. Microbiological Profile of Diabetic Foot Ulcer. Cali Med J; 9 (3):1-4.

14. Chopdekar.K.A, Joshi.A.A, 2011. Bacteriological profile of diabetic foot infection. Bom Hosp J;4 (53):706-11.

15. Kaur. N, Kaur. A, 2014. Clinical and susceptibility profile from diabetic foot patients in tertiary care hospital. Sci J App Med Sci; 2(2): 865-69.

16. Paul. S, Barali. L, 2009. A bacterological study of diabetic foot infection in an urban tertiary care hospital of Dhaka city. Ibrahim Med Coll; 3(2): 50-4.

\section{How to cite this article:}

Greesh, K. and Nepolean, R. 2019. Drug Resistance in an Organisms of Diabetic Foot Patients attending Tertiary Care Hospital in Kulasekharam. Int.J.Curr.Microbiol.App.Sci. 8 (03): 12581268. doi: https://doi.org/10.20546/ijcmas.2019.803.149 